HISTORIA DE LA MEDICINA

\title{
Historia de la dermatología pediátrica en el mundo occidental y en Honduras
}

\author{
History of pediatric dermatology in the western world and in Honduras
}

\section{Gustavo Adolfo Lizardo Castro}

Médico Especialista en Pediatría, Sub-especialista en Dermatología Pediátrica.

Departamento de Pediatría, Servicio de Dermatología, Hospital Escuela; Universidad Nacional Autónoma de Honduras.

\section{INTRODUCCIÓN}

En la mayor parte de los países de Latinoamérica los niños y adolescentes representan el $60 \%$ de la población y en la consulta de un pediatra general entre el $10-20 \%$ de los pacientes presentan lesiones dermatológicas. ${ }^{1}$ Las enfermedades de la piel, congénitas 0 adquiridas, han llamado la atención desde tiempos prehistóricos y existe evidencia escrita en civilizaciones antiguas. La dermatología pediátrica como una subespecialidad, surgió en la segunda mitad del siglo XX es de creación reciente, ${ }^{2}$ como consecuencia por ende es de creación reciente, como consecuencia del desarrollo de dos especialidades, que son sus ancestros, la pediatría encargada de la promoción, prevención de la salud y tratamiento de las enfermedades de los niños y adolescentes y la dermatología, que se encarga del cuidado de la piel en condiciones de salud y enfermedad. El término pediatría se deriva de dos palabras griegas paidós (niños) y iatros (curador); ${ }^{3}$ la palabra dermatología se deriva también de palabras griegas dérma $(\text { piel) })^{4}$ y logos (tratado). Tanto la pediatría como la dermatología en los anales de la historia, son especialidades jóvenes que apenas superan dos siglos de existencia, ${ }^{5,6}$ por lo que su desarrollo forma parte de la evolución histórica de la medicina. En este escrito se relata el desarrollo y evolución de la dermatología pediátrica en el mundo occidental en general y en Honduras en particular.

\section{ANTECEDENTES}

Los Sumerios (Mesopotamia) 5000 años antes de Cristo $(\mathrm{aC})$, específicamente en el recién nacido describieron el vernix caseoso y enfermedades cutáneas congénitas. ${ }^{7}$ Otra evidencia es encontrada en los Papiros de Ebers en Egipto, una enciclopedia médica de más de 3000 años y en los sagrados libros Hindus de la antigua India. ${ }^{7,8}$ Hipócrates (Grecia 460-370 aC), dentro de sus aportes innovadores en muchas

Recibido: 16-03-2020; Aceptado para publicación 31-08-2020

Dirección para correspondencia: Dr. Gustavo Lizardo,

Correo electrónico: glizardoc@yahoo.com

Declaración de relaciones y actividades financieras y no financieras y conflictos de interés: ninguna.

DOI: https://doi.org/10.5377/rmh.v88iSupl.1.11673 áreas de la medicina, en lo concerniente a la dermatología pediátrica existe evidencia relevante, encontrada al hacer un estudio sistemático del Corpus Hippocraticum. Describió varias enfermedades neonatales y pediátricas incluyendo intertrigo, erupciones liquenoides, vitíligo, forúnculo, lepra, enfermedades papuloescamosas, lesiones por picaduras de insectos, exantemas maculares, úlceras asociadas con alopecia, alopecia areata, verrugas, escabiosis, impétigo y bulas, atribuyendo su causa a la flema. Indicó diversos tratamientos como vinagre, azufre, ungüentos, baños al vapor o con agua de mar. En la práctica dermatológica actual el uso de ungüentos específicos y la balneoterapia, son todavía válidos. ${ }^{8,9}$ Rhazes (Persia 865925) describió el eccema infantil y es autor del primer libro pediátrico The Diseases of Children. El primer hospital pediátrico en Europa es el Hopital des Enfants Malades (Hospital para niños enfermos), en Paris en 1802. La dermatología moderna comienza con Robert Willan (Inglaterra 1757-1812) quien publicó en 1808 su libro On Cutaneous Diseases, identificó lesiones primarias y secundarias en su clasificación morfológica de las enfermedades de la piel. ${ }^{6}$ Walter Dendy (Inglaterra 1794-1871), en 1827 publicó el primer libro de dermatología pediátrica Cutaneous Diseases Incidental to Childhood. ${ }^{7}$ Henry Harris Perlman fue el primer dermatólogo pediatra de Estados Unidos, certificado por la Academia Americana de Pediatría y la Academia Americana de Dermatología. Publicó su libro Pediatric Dermatology en $1960 .^{10,11}$

\section{RECONOCIMIENTO GLOBAL COMO SUBESPECIALIDAD}

Durante el Primer Simposio Internacional de Dermatología Pediátrica en octubre de 1973 en Ciudad de México, convocado por el Jefe del Servicio de Dermatología Dr. Ramón Ruiz Maldonado en las instalaciones de la Institución Mexicana de Asistencia a la Niñez (IMAN), ahora Instituto Nacional de Pediatría (INP), con la asistencia de 26 países, entre ellos los pioneros de la dermatología pediátrica de sus respectivos países, la dermatología pediátrica fue "oficialmente nacida". Durante el simposio, en una cena para profesores extranjeros en el restaurante San Angel Inn, a propuesta del Dr. Ruiz Maldonado se fundó con entusiasmo, la Sociedad Internacional de Dermatología Pediátrica. Entre los pioneros presentes se 
encontraban: Martin Beare (Irlanda); Ferdinando Giannotti (Italia); Gabriela Lowy (Brasil); José María Mascaró (España); Edmund Moynahan (Inglaterra); Dagoberto Pierini (Argentina); Lawrence Solomon, Sydney Hurwitz, Alvin Jacobs, Samuel Weinberg, Guinter Khan, Coleman Jacobson y Andrew Margileth (Estados Unidos); Eva Torok (Hungría) y Kasuya Yamamoto (Japón). ${ }^{2,10}$ Desde entonces auspiciado por dicha Sociedad, el Congreso Mundial de Dermatología Pediátrica ha tenido lugar cada tres años, iniciando en 1976 en México, seguido por Chicago, Monte Carlo, Tokio, Milán, Toronto, Buenos Aires, Paris, Cancún, Roma, Tailandia, etc., ${ }^{2,7}$

El interés por esta disciplina se ha incrementado de manera explosiva, resultando atractiva sobre todo para pediatras, dermatólogos y médicos generales, a nivel internacional. Se han fundado numerosas sociedades nacionales y regionales: Estados Unidos, Society for Pediatric Dermatology en 1975, realizando su primer congreso anual en Aspen, Colorado en 1976; Japón, la Japanese Society for Pediatric Dermatology en 1977; Europa, the European Society for Pediatric Dermatology en 1983 y el primer Congreso bianual fue realizado en Munster, Alemania en 1984; América Latina, la Sociedad Latinoamericana de Dermatología Pediátrica en 1996, realizando congresos cada 3 años, a partir de 1997 en Bogotá, Caracas, Lima, Buenos Aires y así sucesivamente. Cursos, Simposios, Congresos y Revistas han sido auspiciadas por sus respectivas Sociedades. Publicaciones de revistas: Japón, Japanese Society for Pediatric Dermatology desde 1982; Estados Unidos, Pediatric Dermatology desde 1983, con alto perfil clínico y de investigación; Europa, European Journal of Pediatric Dermatology comenzó en 1982 como Pediatric Dermatology News, editado por Ernesto Bonifazi; América Latina, Revista de Dermatología Pediátrica Latinoamericana. ${ }^{7}$

Edición de excelentes libros de texto: Sidney Hurwitz, Clinical Pediatric Dermatology; Williams Weston, Dermatología Pediátrica; Lawrence Schachner, Pediatric Dermatology; John Harper, Texbook of Pediatric Dermatology; ${ }^{7}$ Ramón RuizMaldonado, Lawrence Parish y Martin Beare, Texbook of Pediatric Dermatology, en 1989, traducido al español en 1992. Otras publicaciones: Ruiz-Maldonado, Saul, Ibarra, Tamayo, Temas de Dermatología Pediátrica, en 1980; Ruiz-Maldonado, Tamayo Sánchez, Durán McKinster, Orozco Covarrubias, Dermatología en la consulta pediátrica: Diagnóstico y Tratamiento, en 2008; Margarita Larralde, Eugenia Abad, Paula Luna, Dermatología Pediátrica, segunda edición, en 2010.

\section{DERMATOLOGÍA PEDIÁTRICA EN AMÉRICA}

México tiene un programa de residencia en dermatología pediátrica desde 1973, fundado por Dr. Ramón Ruiz-Maldonado y Dra. Lourdes Tamayo, en el Servicio de Dermatología del INP, con duración de 3 años para pediatras certificados y 1 año para dermatólogos certificados, siendo diplomados en dermatología pediátrica. ${ }^{2,12} \mathrm{En}$ la actualidad el total de egresados es de 128: pediatras 80 y dermatólogos 48, siendo 91 mexicanos y 37 extranjeros (Venezuela 10; Colombia 8; El Salvador 4; Perú 3; Guatemala, Panamá, Brasil y Bolivia 2 cada uno; Honduras, Chile, República Dominicana y España 1 cada uno). En 1986 el Dr. Alejandro García Vargas, en Guadalajara, México, estableció un curso de especialización en dermatología pediátrica con duración de 3 años para pediatras y de 1 año para dermatólogos, que incrementó a 3, posteriormente. EI Hospital Infantil de México Federico Gómez, fundó en 1987 un curso de entrenamiento en dermatología pediátrica para pediatras, a cargo de la Dra. Guadalupe Ibarra Durán. A partir de 1997 el Dr. Carlos Mena Cedillos, es profesor titular del Curso con duración de 3 años. Cabe señalar que México fue el primero y por muchos años el único país en contar con este Curso de Especialización. En Guatemala en 1990 el Dr. Edgar Pérez Chavarría y la Dra. Aída Cecilia Pacheco, iniciaron un curso de entrenamiento para pediatras y dermatólogos con duración de 1 año, en el Instituto de Dermatología y Cirugía de Piel (INDERMA), habiendo egresado a la fecha un total de 8, siendo 4 guatemaltecos y 4 hondureños.

En Argentina el Dr. José Antonio Mássimo en 1997 fundó la residencia en dermatología pediátrica en el Hospital Ricardo Gutiérrez y en 1999 la Dra, Margarita Larralde ${ }^{13}$ lo hizo en el Hospital Ramos Mejía, ambos continúan como profesores titulares y está dirigido a pediatras y dermatólogos con duración de 3 años. En la actualidad hay 5 sedes, todas en Buenos Aires con un total de egresados superior a 200. En Venezuela el Dr. Luis Alfredo González Aveledo ${ }^{13}$ de 1993-1995 graduó una única promoción de tres egresados, en Caracas; el Dr. Leopoldo DíazLandaeta y la Dra. Magalis Herrera en el 2005 fundaron un curso capacitación en dermatología pediátrica para pediatras con duración de 3 años, en el Hospital de Especialidades Pediátricas de Maracaibo, pero por razones personales la Dra. Herrera a los pocos meses se retiró del programa, continuando hasta el momento el Dr. Díaz-Landaeta con la titularidad del mismo. En Curitiba, Brasil, la Universidad Federal de Paraná, ofrece un Curso de Especialización en Dermatología Pediátrica para pediatras, de 2 años de duración. En la actualidad prácticamente todos los Cursos de Especialización en Dermatología Pediátrica referidos, han logrado certificación universitaria en sus respectivos países.

En Estados Unidos de América (USA), los pioneros de la dermatología pediátrica son: Carroll Burgoon, Jr., Alvin Jacobs, Sidney Hurwitz, Guinter Kahn, Peter Koblenzer, Arthur Norins, James Rasmussen, Lawrence Solomon, Nancy Esterly, Samuel Weinberg y William Weston. ${ }^{10}$ En el año 2004 la dermatología pediátrica fue certificada como subespecialidad, siendo requisito la certificación previa por el consejo americano de Pediatría y de Dermatología, seguida de la implementación de un programa de recertificación. ${ }^{7,14}$ En 2010, encuestas independientes realizadas por la Asociación Nacional de Hospitales de niños y por la Sociedad para Dermatología Pediátrica, documentaron que el tiempo de espera para una cita de dermatología pediátrica era entre 60-90 días, más larga que cualquier otra subespecialidad pediátrica. A pesar de la demanda, el interés en la subespecialidad ha sido bajo, con solo $2.1 \%$ (233) de dermatólogos pediatras certificados, entre más de 11,000 dermatólogos. ${ }^{14}$ Entre los factores disuasivos para los pediatras, es calificar para una altamente competitiva residencia en dermatología, con duración de 4 años y un 
fuerte impacto financiero ya que la legislación de 1999, limita obtener fondos federales para más de una residencia. Para los dermatólogos sobre todo aquellos con deudas educacionales, las discrepancias en el salario pueden ser decisivas, ya que subespecialidades de procedimientos, cosméticos 0 dermatopatología, el salario es significativamente mayor que el de un dermatólogo pediatra. ${ }^{14}$ Eso explica en parte la escasez de profesionales de la dermatología pediátrica en los hospitales de ese país. En Canadá la dermatología pediátrica ha sido desarrollada por Bernice Krafchik (Toronto), Julie Prendiville (Vancouver), Julie Powell y Danielle Marcoux (Montreal). En 1992, Toronto fue sede del Sexto Congreso Mundial de Dermatología Pediátrica, organizado por Bernice Krafchik y James Rasmussen (Buffalo, New York, USA).?

En realidad, la oferta de dermatólogos pediatras en el mundo occidental, o sea en Europa, Estados Unidos, Canadá y Latinoamérica es escasa, en parte por tratarse de una disciplina relativamente nueva, con pocos centros académicos dedicados a la formación de dichos profesionales, razón por la cual en Latinoamérica hay muchos países que en la actualidad no cuentan con ningún dermatólogo pediatra.

\section{DERMATOLOGÍA PEDIÁTRICA EN HONDURAS}

En Honduras al igual que muchos países, las enfermedades de la piel en niños y adolescentes han sido tradicionalmente manejadas por pediatras, quienes tienen escasa formación dermatológica o por dermatólogos generales que en ocasiones se encontraban con genodermatosis o exantemas que nunca habían visto en adultos, por lo que eventualmente el resultado fue poco satisfactorio, en vista de su inadecuada preparación en la fisiopatología, diagnóstico y tratamiento de los desórdenes cutáneos en niños y adolescentes. Poco a poco se fue aceptando la conveniencia de que valía la pena tener un dermatólogo pediatra para el manejo de las enfermedades de la piel en este grupo etario. ${ }^{12}$

En junio de 1986 regresó a Tegucigalpa, Honduras, el Dr. Gustavo Adolfo Lizardo Castro, egresado del INP de México, como Especialista en Pediatría y Sub-especialista en Dermatología Pediátrica (incluye una rotación por 3 meses en el Children's Hospital of Philadelphia, con el dermatólogo pediatra Paul J. Honig), siendo el primero en Honduras con esta Subespecialidad. En septiembre de ese año, inició labores con la Universidad Nacional Autónoma de Honduras (UNAH) como profesor titular III en el Departamento de Pediatría. Incorporó la asignatura de dermatología pediátrica al programa académico de $\mathrm{V}$ año de la Carrera de Medicina, desempeñándose en ese cargo hasta el año 2011. A partir del 20 de diciembre del 2011 fue nombrado Coordinador de Investigación primero y luego Coordinador Académico del Posgrado de Pediatría hasta el 2017. Desde 1986 incorporó al tercer año del Posgrado de Pediatría el módulo de dermatología pediátrica, mismo que desempeñó hasta el año 2017 inclusive.

En noviembre de 1986 el Dr. Lizardo inició labores en el Hospital Escuela (HE), Secretaría de Salud, asignado como Jefe de la Sala de Emergencia de Pediatría por el Dr. Francisco Cleaves Tomé, entonces jefe del Departamento de Pediatría. En enero de 1994, gracias a la gestión de la Dra. Ofelia Wilkinson Oberti, Jefa del Servicio de Dermatología del HE, fue reubicado a dicho Servicio como encargado de la Consulta Externa de Dermatología Pediátrica e Interconsultas. La demanda fue considerable con más de 3000 consultas anuales, aportando al mismo tiempo docencia y práctica supervisada al Posgrado de Dermatología, hasta su jubilación como médico asistencial en marzo del 2014. A partir de entonces continuó desempeñándose como docente de la UNAH en la capacitación teórico-práctica en dermatología pediátrica de los residentes del Posgrado de Dermatología, hasta la actualidad (Figura 1). La consulta dermatológica pediátrica fue reforzada con la Dra. Verónica Meléndez Oviedo, dermatóloga pediatra, de marzo del 2006 a octubre de 2014, siendo trasladada al Hospital María de Especialidades Pediátricas (HMEP), para continuar sus labores en dicha institución.

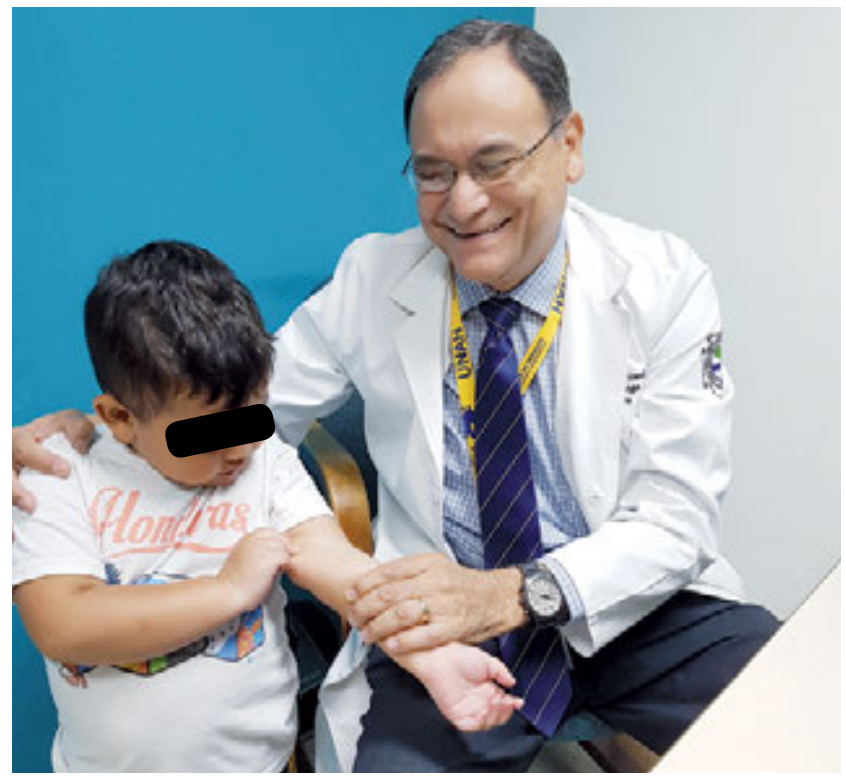

Figura 1. El Dr. Gustavo Lizardo con paciente de 3 años de edad, quien le señala una pequeña lesión de molusco contagioso, Consulta Externa del Servicio de Dermatología, Hospital Escuela, Tegucigalpa.

Los dermatólogos pediatras certificados por la UNAH y el Colegio Médico de Honduras son seis: Dr. Gustavo Adolfo Lizardo Castro, Especialista en Pediatría, egresado del INP, México y Sub-especialista en Dermatología Pediátrica, egresado del INP, México en 1986, Profesor Titular, Dr. Ramón Ruiz Maldonado y Dra. Lourdes Tamayo Sánchez. Trabaja como docente del Posgrado de Dermatología de la UNAH, en Tegucigalpa; Dr. Héctor Caballero Castro, Especialista en Pediatría, egresado del Hospital San Juan de Dios, Guatemala y Sub-especialista en Dermatología Pediátrica, egresado del INDERMA, Guatemala en 1996, Profesor Titular Dr. Edgar Pérez Chavarría y Dra. Aída Pacheco. Trabaja en el Instituto Hondureño de Seguridad Social, San Pedro Sula y es docente de la UNAH Valle de Sula; Dr. Carlos Anariva Cálix, Especialista en Pediatría, egresado del Posgrado de Pediatría, UNAH y Sub-especialista en Dermatología Pediátrica, egresado del INDERMA, Guatemala en 2000, Profesor Titular, Dr. Edgar 
Pérez Chavarría y Dra. Aída Pacheco. Reside en la Ciudad de El Progreso, Yoro; Dra. Verónica Isabel Meléndez Oviedo, Especialista en Dermatología, egresada del Posgrado de Dermatología, UNAH y Sub-especialista en Dermatología Pediátrica, egresada del Instituto Dermatológico de Jalisco, Guadalajara, Jalisco, México en 2006, Profesor Titular Dr. Alejandro García Vargas. Trabaja en el HMEP y es docente de Grado de Medicina de la Universidad Católica en Tegucigalpa; Dra. Ada Victoria Argueta Espino, Especialista en Pediatría, egresada del Hospital Roosevelt, Guatemala y Sub-especialista en Dermatología Pediátrica, egresada del Hospital Infantil de México Federico Gómez, México en 2008, Profesor Titular Dr. Carlos Mena Cedillos. Trabaja en el Hospital Católico "Hermano Pedro" y tiene su sede en Catacamas, Olancho. Dra Iris Rosbinda Álvarez Montiel, Especialista en Pediatría, egresada del Hospital San Juan de Dios, Guatemala, y Sub-especialista en Dermatología Pediátrica, egresada del INDERMA Guatemala en 2006, Profesor Titular Dr. Edgar Pérez Chavarría y Dra. Aída Pacheco y del Hospital Infantil Federico Gómez, México en 2012, Profesor Titular Dr. Carlos Mena Cedillos. Trabaja como docente de Grado de Medicina y Posgrado de Pediatría de la UNAH en Tegucigalpa, continuando la enseñanza de la dermatología pediátrica iniciada por el Dr. Lizardo.

La dermatología pediátrica ha tenido presencia activa en los congresos nacionales de la Sociedad Hondureña de Dermatología y de la Asociación Pediátrica Hondureña con la presentación por parte de los dermatólogos pediatras, dermatólogos generales y residentes de dermatología, mediante conferencias y presentación de casos clínicos de enfermedades cutáneas de niños y adolescentes de interés para el médico general, pediatras y dermatólogos. En mayo del 2018 tuvo lugar en San Pedro Sula, el Primer Congreso Nacional de Dermatología Pediátrica, "Dr. Gustavo Lizardo" (Figura 2), organizado por la Sociedad Hondureña de Dermatología, Capítulo Nor-occidental, con la participación activa y entusiasta de dermatólogos, pediatras, residentes de dermatología, pediatría y médicos generales, como conferencistas o asistentes. En mayo-junio del 2019, se efectuó el Segundo Congreso anual en Tegucigalpa, siendo San Pedro Sula la próxima sede. De igual manera la dermatología pediátrica hondureña ha tenido participación activa en congresos internacionales organizados por sociedades regionales sobre todo por la Sociedad Centroamericana y del Caribe de Dermatología (SCCAD), el Colegio Iberolatinoamericano de Dermatología (CILAD) y la Sociedad Latinoamericana de Dermatología Pediátrica (SLADP).

Temas relacionados con la dermatología pediátrica de autores hondureños han sido publicados en revistas médicas nacionales (o internacionales), como la Revista Honduras Pediátrica, Revista de la Facultad de Ciencias Médicas; sin embargo, la Revista Médica Hondureña (RMH), es la que cuenta con la mayor parte de las publicaciones de las últimas décadas, sobre la diversidad patológica manifestada en la piel, pelo y uñas de niños y adolescentes. Los trabajos publicados tienen como autores médicos asistenciales y docentes del Servicio de Dermatología del HE, con participación de residentes del Posgrado de Dermatología de la UNAH. Se solicitó la

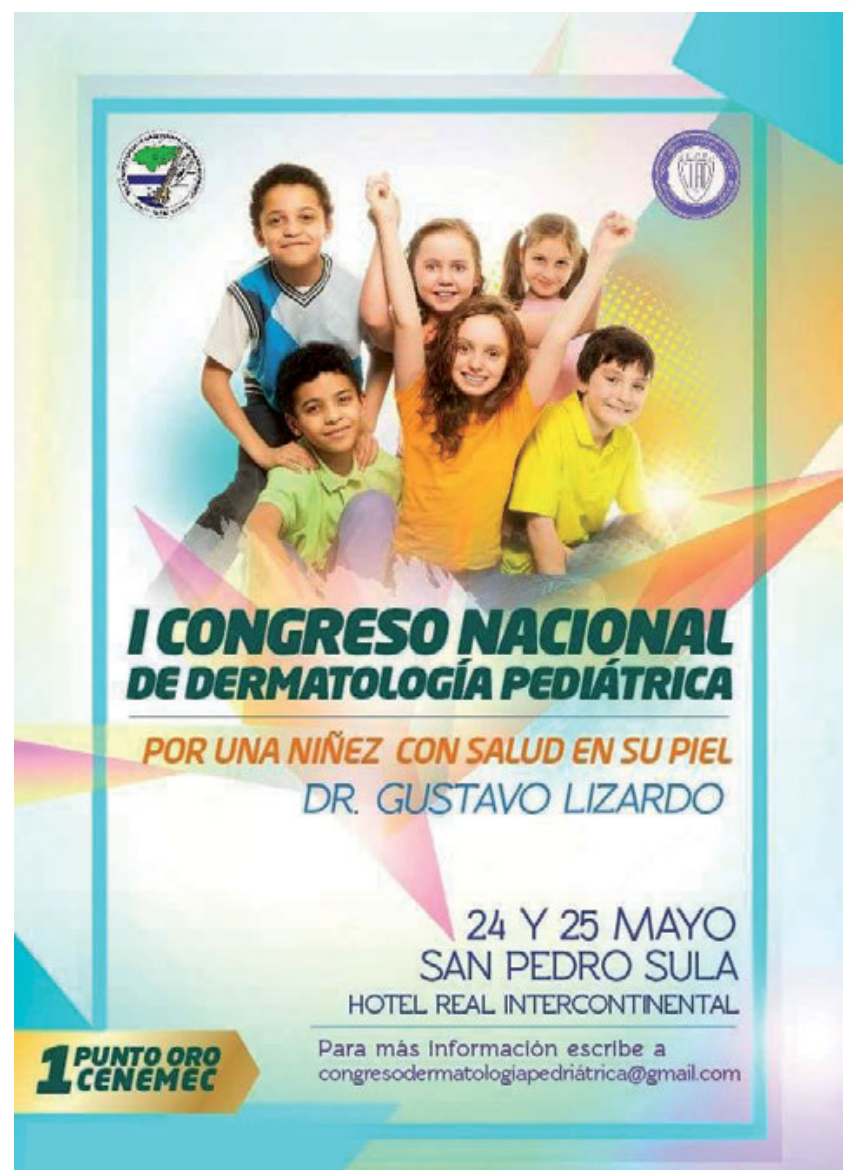

Figura 2. Afiche promocional del Primer Congreso Nacional de Dermatología Pediátrica en la Ciudad de San Pedro Sula, Honduras, mayo del año 2018.

colaboración de la Biblioteca Médica Nacional para realizar la búsqueda de las publicaciones en la RMH de los dermatólogos y pediatras. A continuación, se describen publicaciones, categorizadas por etiología:

\section{A. Enfermedades infecciosas}

1. Lizardo Castro G, Ponce C, Ponce E, Sierra M. Caracterización de pacientes con leishmaniasis cutánea atípica. Rev Med Hondur 2008;76(3):101-7.

2. Matute N, Espinoza C, Alger J, Padgett D, López E, Zúniga C. Caracterización clínico-epidemiológica de pacientes con leishmaniasis atendidos en el Hospital Escuela. Rev Med Hondur 2009;77(1):7-15.

3. Martel B, Corrales Padilla H, Ponce C, Ponce E. Criocirugía como alternativa terapéutica en leishmaniasis cutánea atípica. Rev Med Hondur 1997;65(2):62-4.

4. Lizardo Castro G, Gómez Campos A. Larva migrans cutánea. Rev Med Hondur 2019;87(2):84.

5. Mejía de Calona A, de Sierra O, Navarro F. Micosis superficiales en el Hospital Escuela. Rev Med Hondur 1991; 59:8-13.

6. Lizardo Castro G, Lizardo AE. Presentación inusual de onicomicosis por Candida albicans. Rev Med Hondur 2012;80(2):61-5. 
7. Lizardo Castro G, Velásquez D. Candidiasis miliar por pañal. Rev Med Hondur 2015;83(1,2):55.

8. Lizardo Castro G, Zúniga Muñoz P. Esporotricosis linfocutánea en niños: a propósito de un caso. Rev Med Hondur 2011;79(2):81-4.

9. Lizardo Castro G, García D. ¿Qué debemos saber hoy en día de la fascitis necrotizante? A propósito de dos casos. Rev Med Hondur 2011;79(4):203-6.

10. López Lutz E. Linfogranuloma venéreo. Rev Med Hondur 2000;68:15.

B. Trastornos genéticos

11. Durón RM, Lizardo G, López Lutz E, Morales S, Hesse $H$, Molina L, et al. Síndromes neurocutáneos en la consulta neurológica: serie de casos. Rev Med Hondur 2009;77(4):172-6.

12. Matute N, Meléndez V. Ectrodactilia-Displasia ectodérmica-Labio/Paladar hendido: reporte de un caso. Rev Med Hondur 2007;75:26-9.

\section{Trastorno autoinmune}

13. González M, Ortiz Paredes K, Sandoval NJ. Lupus eritematoso cutáneo subagudo: reporte de caso y revisión de la literatura. Rev Med Hondur 2008;76:114-7.

\section{Anomalía vascular}

14. Lizardo Castro G, Velásquez DG, Cruz Enamorado B. Hemangioma congénito rápidamente involutivo. Rev Med Hondur 2015;83(3,4):130-3.

\section{E. Causa desconocida}

15. Ortiz Paredes K, Meléndez Oviedo V. Atrofodermia de Pasini y Pierini unilateral. Rev Med Hondur 2010;78(2):80-2.

16. Lizardo Castro G, García DM. Liquen estriado. Rev Med Hondur 2011;79(2):74.

Los primeros tres trabajos corresponden a Leishmaniasis, enfermedad tropical desatendida, transmitida por vector, es endémica en Honduras, sobre todo la variante cutánea atípica en la región sur, descrita por el Dr. Ponce et al..$^{15}$ y que afecta principalmente a niños entre 5 y 15 años. Los artículos de los numerales 2, 5 y 11, la población estudiada correspondió a niños y adultos. El numeral 11 además, fue un estudio colaborativo del Posgrado de Neurología de la UNAH con el Servicio de Dermatología del HE y por consiguiente ambos, aportaron los pacientes participantes.

\section{CONCLUSIÓN}

La dermatología pediátrica siendo una disciplina muy joven con apenas 47 años de haber nacido oficialmente, ha tenido un amplio reconocimiento nacional e internacional y se ha consolidado como una subespecialidad con fuerza propia, respaldada por avances científicos y tecnológicos que han abierto el horizonte para la investigación, epidemiología, conocimiento, diagnóstico y manejo de la diversa y compleja patología cutánea de niños y adolescentes. A diferencia de muchos países de Latinoamérica y de varias ciudades de Estados Unidos, que no tienen acceso a un profesional de la dermatología pediátrica, en Honduras se cuenta con los servicios de dermatólogos pediatras en varias regiones del país. Un desafío para Latinoamérica y el mundo es crear las condiciones apropiadas para la formación del recurso humano necesario para satisfacer las necesidades de la población en el campo de la dermatología pediátrica.

\section{AGRADECIMIENTO}

Se agradece la colaboración de la Biblioteca Médica Nacional por haber proporcionado las publicaciones en la Revista Médica Hondureña de los dermatólogos y pediatras. Se agradece la información proporcionada por: Servicio de Dermatología, Instituto Nacional de Pediatría, México; Dr. Carlos Mena Cedillos, Hospital Infantil de México Federico Gómez; Dr. Edgar Pérez Chavarría, INDERMA, Guatemala; Dr. Luis Alfredo González Aveledo, Caracas, y Dr. Leopoldo Díaz Landaeta, Hospital de Especialidades Pediátricas, Maracaibo, Venezuela; Dra. Margarita Larralde, Hospital Ramos Mejía, Dr. José Antonio Mássimo, Hospital Dr. Ricardo Gutiérrez y Dra. Graciela Manzur, Buenos Aires, Argentina.

\section{REFERENCIAS}

1. Ruiz Maldonado R, Tamayo Sánchez L, Durán McKinster C, Orozco Cobarrubias ML. Dermatología en la Consulta Pediátrica: Diagnóstico y Tratamiento. México: ETM; 2008.

2. Ruiz-Maldonado R. Historia de la Dermatología Pediátrica en México. En: Galimberti R, Pierini AM, Cervini AB, eds. Historia de la Dermatología Latinoamericana. Toulouse: Editions Privat; 2007. p. 273-6.

3. Smith Y. A Brief History of Pediatrics. [Internet]. Manchester: NewsMedical; 2019. [consultado 25 junio 2020]. Disponible en: https://www. news-medical.net/health/A-Brief-History-of-Pediatrics.aspx

4. Santoro R. Skin over the Centuries. A short history of dermatology: physiology, pathology and cosmetics. Medhistór. 2017;1(2):94-102.

5. Luecke Jr PE. The History of Pediatrics at Baylor University Medical Center. Proc (Bayl Univ Med Cent) .2004;17(1):56-60.

6. Ross JB. The origins of Western Dermatology. J Cutan Med Surg. 2010;14(6):255-66.

7. Harper J. The History of Paediatric Dermatology. In: Irvine AD, Hoeger PH, Yan AC, eds. Harper's Texbook of Pediatric Dermatology. Philadelphia: John Wiley; 2011.p 1.1-1.5.

8. Rabdill SX. Pediatric Dermatology in Antiquity: Part 1. Int J Dermatol
1975;14(5):363-8.

9. Sgantzos M, Tsoucalas G, Karamanou M, Giatsiou S, Tsoukalas I, Androutsos G. Hippocrates on Pediatric Dermatology. Pediatr Dermatol 2015;32(5):600-3.

10. Hurwitz $\mathrm{S}$. The History of Pediatric Dermatology in the United States. Pediatr Dermatol. 1988;5(4):280-5.

11. Moynahan EJ. Book Review Paediatric Dermatology. Br Med J 1961;1:954.

12. Ruiz-Maldonado R. Pediatric Dermatology Accomplishments and Challenges for the 21st Century. Arch Dermatol. 2000;136(1):84.

13. Halpert $\mathrm{E}$, Ruíz-Maldonado R, Cáceres $\mathrm{H}$. Desarrollo de la dermatología pediátrica en América Latina. En: Galimberti R, Pierini AM, Cervini AB, eds. Historia de la Dermatología Latinoamericana. Toulouse: Editions Privat;2007. p. 449-50.

14. Prindaville B, Antaya RJ, Siegfried EC. Pediatric Dermatology: Past, Present, and Future. Pediatr Dermatol 2015;32(1):1-12.

15. Ponce C, Ponce E, Morrison A, Cruz A, Kreutzer R, McMahon Pratt D, et al. Leishmania donovani chagasi: new clinical variant of cutaneous leishmaniasis in Honduras. Lancet. 1991:12;337(8733):67-70. 